\title{
Neutrino fluxes from dark matter in the HESS J1745-290 source at the Galactic center
}

\author{
J. A. R. Cembranos, ${ }^{1,2, *}$ V. Gammaldi, ${ }^{2, \dagger}$ and A. L. Maroto, ${ }^{2, *}$ \\ ${ }^{1}$ SLAC National Accelerator Laboratory, 2575 Sand Hill Road, Menlo Park, California 94025, USA \\ ${ }^{2}$ Departamento de Física Teórica I, Universidad Complutense de Madrid, E-28040 Madrid, Spain
} (Received 29 April 2014; published 7 August 2014)

\begin{abstract}
The spectral study of the HESS J1745-290 high-energy gamma-ray cutoff from the Galactic center is compatible with a signal of dark matter (DM) annihilation or decay. If this is the case, a neutrino flux from that source is also expected. We analyze the neutrino flux predicted by DM particles able to create the HESS J1745-290 gamma-rays observations. We focus on the electroweak and hadronic channels, which are favored by present measurements. In particular, we study DM annihilating into $W^{+} W^{-}$and $u \bar{u}$ with DM masses of 48.8 and $27.9 \mathrm{TeV}$, respectively. We estimate the resolution angle and exposition time necessary to test the DM hypothesis as the origin of the gamma-ray signal.
\end{abstract}

DOI: $10.1103 /$ PhysRevD.90.043004

\section{INTRODUCTION}

Different telescopes have observed very-high energy (VHE) gamma rays coming from the Galactic center (GC), such as CANGAROO [1], VERITAS [2], MAGIC [3], and Fermi-LAT [4,5]. In this work, we will pay attention to the data collected by the HESS collaboration from the J1745-290 source during the years 2004-2006 $[6,7]$. The variability of the IR and x-ray observations [8] indicates a different emission mechanism for this part of the spectrum. In addition, one of the most characteristic features of the HESS J1745-290 data consists in a cutoff at several tens of TeVs. These spectral properties can be explained naturally by the photons produced by the annihilation or decay of dark matter (DM) particles. This interpretation was discussed from the very early days of the publication of the HESS data $[9,10]$ but it was concluded that the DM origin was disfavored [10]. However, a recent study has shown that the observed data are well fitted by a DM signal complemented by a diffuse background [11]. Indeed, this background has a good motivation since VHE photons are also expected from radiative processes generated by particle acceleration in the neighborhood of the supermassive black hole Sgr A and the Sgr A East supernova. The analysis shows good agreement with DM annihilation or decay into $u \bar{u}, d \bar{d}, s \bar{s}$, and $t \bar{t}$ quarkantiquark channels and $W^{+} W^{-}$and $Z Z$ boson channels. Leptonic and other quark-antiquark channels were excluded at the $95.4 \%$ confidence level. The background provided by the analysis is also compatible with the FermiLAT data from the IFGL J1745.6-2900 source observed during 25 months [5], which is spatially consistent with the HESS J1745-290 source [12].

\footnotetext{
cembra@slac.stanford.edu

vivigamm@ucm.es

¥maroto@fis.ucm.es
}

In any case, the fundamental nature of this gamma-ray flux is still unclear. The entire VHE spectrum may be produced by particle propagation $[5,13]$ in the vicinity of the Sgr A supernova remnant and black hole, both located at the central region of our Galaxy [14,15]. In addition, the emission region is quite compact since the signal is limited to a region of a few tenths of a degree [7]. This feature is not consistent with dark halos simulated with nonbaryonic cold DM, such as the standard Navarro-Frenk-White (NFW) profile [16]. It needs to be more compact like those produced when baryonic effects are taken into account. It has been argued that the baryonic gas falls to the inner part of the halo, modifying the gravitational potential and increasing the DM density in the center $[17,18]$. This scenario is not completely accepted (read Ref. [19], for example), but if it is correct, it has two important consequences. First, the sensitivity of indirect DM searches is reduced to a more compressed region, and second, the DM annihilating fluxes are enhanced by up to 3 orders of magnitude with respect to the standard NFW profile [18]. The HESS observations are in good agreement with these types of compressed dark halos.

The DM particle that creates this spectrum needs to have a mass between $15 \mathrm{TeV} \lesssim M \lesssim 110 \mathrm{TeV}$ [11]. This makes it highly challenging to observe these particles in direct-detection experiments or particle accelerators [20]. On the other hand, complementary cosmic-ray analyses [21] are the most promising way to cross-check these DM hypotheses.

In particular, the analysis of neutrino fluxes from the same region can be definitive. If DM annihilates or decays into Standard Model (SM) particles producing VHE gamma-ray photons, it also has to produce VHE neutrinos. Indeed, if the dark halo properties are adjusted to explain the HESS J1745-290 data, the neutrino flux is completely determined if one concrete annihilation or decay channel is 
assumed. This work is organised as follows. In Sec. II, we study the expected neutrino fluxes as indirect products of annihilating DM in the direction of the GC. Section III is devoted to discussing the flavor-oscillation effects in this signal. In Sec. IV, we model the background of our analysis by taking into account the atmospheric neutrino flux observed by the IceCube experiment and we study the best configuration that may allow for the detection of the corresponding neutrino signal associated with the HESS J1745-290 GC gamma-ray source. Finally, we summarize our main conclusions in Sec. V.

\section{ASTROPHYSICAL NEUTRINO FLUX}

The differential flux of neutrinos of a given flavor $\nu_{f}$ observed at the Earth in a particular direction can be computed as

$$
\begin{aligned}
\frac{d \Phi_{\nu_{f}}}{d E}= & \sum_{p=1}^{3} \sum_{a=1}^{2} \sum_{i}^{\text {channels }} P_{f p} \cdot \frac{\zeta_{i}^{\left(a, \nu_{p}\right)}}{a} \\
& \times \frac{d N_{i}^{\left(\nu_{p}\right)}}{d E} \cdot \frac{\left\langle J_{(a)}\right\rangle_{\Delta \Omega}}{4 \pi M^{a}}
\end{aligned}
$$

where $P_{f p}$ are the elements of the symmetric $3 \times 3$ matrix which takes into account the neutrino-oscillation effects from the produced neutrino flavor $\left(\nu_{p}\right)$ generated by the DM from Galactic sources to the observed neutrino flavor $\left(\nu_{f}\right)$ at the Earth. We shall discuss these effects in detail in the next section. $M$ is the mass of the DM particle. The case $a=2$ accounts for neutrinos coming from DM annihilation with $\zeta_{i}^{\left(2, \nu_{p}\right)} \equiv\left\langle\sigma_{i}^{\nu_{p}} v\right\rangle$ being the thermal averaged annihilation cross section of two DM particles (assumed to be their own antiparticles) into SM particles (also labeled by the subindex $i$ ). If $\mathrm{DM}$ is metastable, neutrinos can also be produced by its decay. In such a case the contribution with $a=1$ is activated, with $\zeta_{i}^{\left(1, \nu_{p}\right)} \equiv \Gamma_{i}^{\nu_{p}}$ being the decay width into SM particles (labeled by the same subindex $i$ ).

The number of neutrinos of flavor $\nu_{p}$ produced in each annihilating or decaying channel $d N_{i}^{\left(\nu_{p}\right)} / d E$ involves the decay and/or hadronization of unstable products, such as quarks and leptons. Because of the nonperturbative QCD effects, this requires Monte Carlo event generators [22], or fitting or interpolation functions [23]. In particular, we will use the results reported in Ref. [24]. They referred to the PYTHIA 8.135 Monte Carlo event-generator software [22] and reproduced the differential number of neutrinos produced by DM of different masses. In this work, we will focus on neutrino fluxes coming from fragmentation and decays of SM particle-antiparticle pairs produced by DM annihilation. We shall ignore DM decays, the possible production of monoenergetic neutrinos, $n$-body annihilations (with $n>2$ ), or neutrinos produced from electroweak bremsstrahlung. In particular, we will consider DM annihilation into single channels of SM particle-antiparticle pairs that are consistent with the origin of the HESS J1745290 gamma-ray observations, as we have explained.

The DM spatial distribution is encoded in the astrophysical factors $\left\langle J_{(a)}\right\rangle$, which depend on the $\Psi$ angle, determined by the line of observation with respect to the direction of the GC, and the total angular field of view $\Delta \Omega$,

$$
\left\langle J_{(a)}\right\rangle=\frac{1}{\Delta \Omega} \int_{\Delta \Omega} \mathrm{d} \Omega \int_{0}^{l_{\max }(\Psi)} \rho^{a}[r(l)] d l(\Psi),
$$

where $l$ is the distance from the Sun to a particular point of the DM halo, which is related to the radial distance $r$, computed with respect to the GC, through the equation $r^{2}=l^{2}+D_{\odot}^{2}-2 D_{\odot} l \cos \Psi$. The distance between the Sun and the center of the Galaxy is denoted by $D_{\odot} \simeq 8.5 \mathrm{kpc}$, and the maximum distance between the Sun and the edge of the halo in a given direction $\Psi$ is $l_{\max }=D_{\odot} \cos \Psi+$ $\sqrt{r_{\text {max }}^{2}-D_{\odot}^{2} \sin \Psi}$. The differential astrophysical factor is proportional to $\rho^{2}$ when it accounts for DM annihilation and proportional to $\rho$ when it computes a DM decay.

As we have commented, the neutrino fluxes have to be averaged over the field of view of the detector, which we shall parametrize with the angle $\theta: \Delta \Omega=2 \pi(1-\cos \theta)$. The HESS Cherenkov telescope array can be characterized typically by $\Delta \Omega_{\mathrm{HESS}} \simeq 10^{-5}$ or $\theta_{\mathrm{HESS}} \simeq 0.1^{\circ}$. This angular resolution is not precise enough to resolve the J1745-290 gamma-ray morphology, which can be approximated by a point-like source.

It is interesting to note that 0.1 degrees or $10 \mathrm{pc}$ is the maximum order of magnitude of the size of the source to be consistent with the HESS data. On the other hand, the Schwarzschild radius of the central black hole Sagittarius $\mathrm{A}^{*}$ is of the order of $10^{-3} \mathrm{pc}$. Below this distance, the DM density vanishes [25] and below $0.1 \mathrm{pc}$, approximately, the gamma-ray production can be importantly attenuated with the variable IR emission from the Galactic center. However, the majority of the allowed emission volume is not affected by this effect. Indeed, this is due to the fact that this effect will be present although the origin of the studied gamma rays is different from DM annihilation.

Therefore, the integration along the line of sight can be approximated by a constant value for $\theta \gtrsim 0.1^{\circ}$, and the astrophysical factor given by Eq. (2) is fixed by fitting the HESS data,

$$
\left\langle J_{(a)}\right\rangle=\left\langle J_{(a)}\right\rangle_{\mathrm{HESS}} \frac{\Delta \Omega_{\mathrm{HESS}}}{\Delta \Omega},
$$

where $\left\langle J_{(a)}\right\rangle_{\text {HESS }}$ is the astrophysical factor which reproduces the J1745-290 gamma-ray flux, which depends on the particular annihilating or decaying DM channel [11]. Therefore, for a neutrino telescope with $\Delta \Omega \gtrsim 10^{-5}$ the total astrophysical factor $\left(\left\langle J_{(a)}\right\rangle \Delta \Omega\right)$ is constant, whereas the average $\left(\left\langle J_{(a)}\right\rangle\right)$ decreases inversely with $\Delta \Omega$. In 
particular, we will focus on the $W^{+} W^{-}$and $u \bar{u}$ annihilation channels with the standard thermal value $\langle\sigma v\rangle=$ $3 \times 10^{-26} \mathrm{~cm}^{3} \mathrm{~s}^{-1}$. By taking into account the results of Ref. [11],

$$
\left\langle J_{(2)}^{W^{+} W^{-}}\right\rangle=\frac{(7.9 \pm 1.9) \times 10^{22}}{1-\cos \theta} \mathrm{GeV}^{2} \mathrm{~cm}^{-5},
$$

and

$$
\left\langle J_{(2)}^{u \bar{u}}\right\rangle=\frac{(4.4 \pm 0.8) \times 10^{22}}{1-\cos \theta} \mathrm{GeV}^{2} \mathrm{~cm}^{-5}
$$

\section{NEUTRINO FLAVORS AND MIXING}

After simulating the neutrino fluxes produced at the source, one has to take into account different aspects in order to estimate the expected flux as observed at the Earth, such as neutrino oscillations and detector sensitivity to neutrino flavors. On the other hand, we shall assume that our detector is not able to discriminate between neutrinos and antineutrinos. Due to neutrino oscillations, the ratios of neutrino flavors change during the transit from the source to the observer [26]. By considering the standard three-flavor neutrino oscillation, the probability matrix $P$ for astrophysical neutrinos traversing a vast distance is given by

$$
P(i \rightarrow j)=\sum_{a=1}^{3}\left|U_{i a}\right|^{2}\left|U_{j a}\right|^{2},
$$

where $U_{i a}$ are the elements of the neutrino mixing matrix [27]. For example, for the simplified case of the oscillation between only two flavors at a distance $x$ from the source, the probability can be written as

$$
P(i \rightarrow j)=\sin ^{2}\left(2 \alpha_{i j}\right) \times \sin ^{2}\left(\pi \frac{x}{L}\right) .
$$

It depends on the mixing angle $\alpha$ and the oscillation length $L=4 \pi E / \Delta m^{2} \sim 1 \mathrm{AU} \times(E / \mathrm{TeV}) /\left(\Delta m^{2} / 10^{-4} \mathrm{eV}^{2}\right)$, where $E$ is the energy and $\Delta m^{2} \equiv\left|m_{1}^{2}-m_{2}^{2}\right|$ is the squaredmass difference between the two mass eigenstates. By taking into account that $\Delta m_{21}^{2}=(7.50 \pm 0.20) \times 10^{-5} \mathrm{eV}^{2}$, and $\Delta m_{32}^{2}=2.32_{-0.08}^{+0.12} \times 10^{-3} \mathrm{eV}^{2}$ [28], we can claim that the oscillation length $L$ is of the order of AUs-much smaller than the linear dimension of the source-so that the source is flavor coherent and the oscillations will be averaged out over both dimension and energy. In any case, due to the large distance of the GC with respect to the dimensions of the detector, this fact does not affect the computation [26]. For a point-like source localized in the GC, we can assume that the totally averaged oscillations among the three flavors is given by a symmetric matrix of the form

$$
\left(\begin{array}{l}
\Phi_{\nu_{e}} \\
\Phi_{\nu_{\mu}} \\
\Phi_{\nu_{\tau}}
\end{array}\right)=\left(\begin{array}{lll}
P_{e e} & P_{e \mu} & P_{e \tau} \\
P_{e \mu} & P_{\mu \mu} & P_{\mu \tau} \\
P_{e \tau} & P_{\mu \tau} & P_{\tau \tau}
\end{array}\right)\left(\begin{array}{c}
\Phi_{\nu_{e}}^{0} \\
\Phi_{\nu_{\mu}}^{0} \\
\Phi_{\nu_{\tau}}^{0}
\end{array}\right) .
$$

The elements $P_{\alpha \beta}$ depend on the three mixing angles $\alpha_{i j}$ and the $C P$ phase $\delta$ (see, for example, Ref. [27]). There are important uncertainties associated to these values, but a good and simple approximation is given by assuming $\sin ^{2}\left(2 \alpha_{13}\right)=0$ and $\sin ^{2}\left(2 \alpha_{23}\right)=1$ [the present experimental observations constrain these angles as $\sin ^{2}\left(2 \alpha_{13}\right)=$ $0.095 \pm 0.010$ and $\sin ^{2}\left(2 \alpha_{23}\right)>0.95$ [28]]. In such a case, $P_{\alpha \beta}$ depends only on the $\alpha_{12}$ angle in the following way: $P_{e e} \simeq 1-\sin ^{2}\left(2 \alpha_{12}\right) / 2, \quad P_{e \mu} \simeq P_{e \tau} \simeq 1-\sin ^{2}\left(2 \alpha_{12}\right) / 4$, $P_{\mu \mu} \simeq P_{\mu \tau} \simeq P_{\tau \tau} \simeq 1-\sin ^{2}\left(2 \alpha_{12}\right) / 8$.

This means that the astrophysical fluxes of $\nu_{\mu}$ and $\nu_{\tau}$ are approximately the same, independent of the flavor composition of neutrinos produced at the source. In addition, as the value of $\alpha_{12}$ is important $\left[\sin ^{2}\left(2 \alpha_{12}\right)=0.857 \pm 0.024\right.$ [28]], the oscillation effects need to be taken into account. In any case, as can be seen in Figs. 1 and 2 for the $W^{+} W^{-}$ annihilation channel, we have checked that the neutrino flavor ratios of the fluxes observed at the Earth are very homogeneous: $\Phi_{\nu_{e}}: \Phi_{\nu_{\mu}}: \Phi_{\nu_{\tau}} \simeq 1: 1: 1$. The reason for this is that the majority of the neutrinos come from the charged pion decay chain, $\pi^{+} \rightarrow \mu^{+}+\nu_{\mu} \rightarrow e^{+}+\nu_{\mu}+\nu_{e}+\bar{\nu}_{\mu}$ (or $\pi^{-} \rightarrow \mu^{-}+\bar{\nu}_{\mu} \rightarrow e^{-}+\bar{\nu}_{\mu}+\bar{\nu}_{e}+\nu_{\mu}$ ), which gives an original ratio of $\Phi_{\nu_{e}}^{0}: \Phi_{\nu_{\mu}}^{0}: \Phi_{\nu_{\tau}}^{0} \simeq 1: 2: 0$. This production is dominant except for the mentioned $W^{+} W^{-}$channel at very high energies, where the neutrinos are produced directly by the leptonic decay of the gauge bosons, $W^{+} \rightarrow$ $l^{+}+\nu_{l}$ (or $W^{-} \rightarrow l^{-}+\bar{\nu}_{l}$ ), but this implies that even the original neutrino flux produced by the source is already homogeneous: $\Phi_{\nu_{e}}^{0}: \Phi_{\nu_{\mu}}^{0}: \Phi_{\nu_{\tau}}^{0} \simeq 1: 1: 1$. In both cases, it is

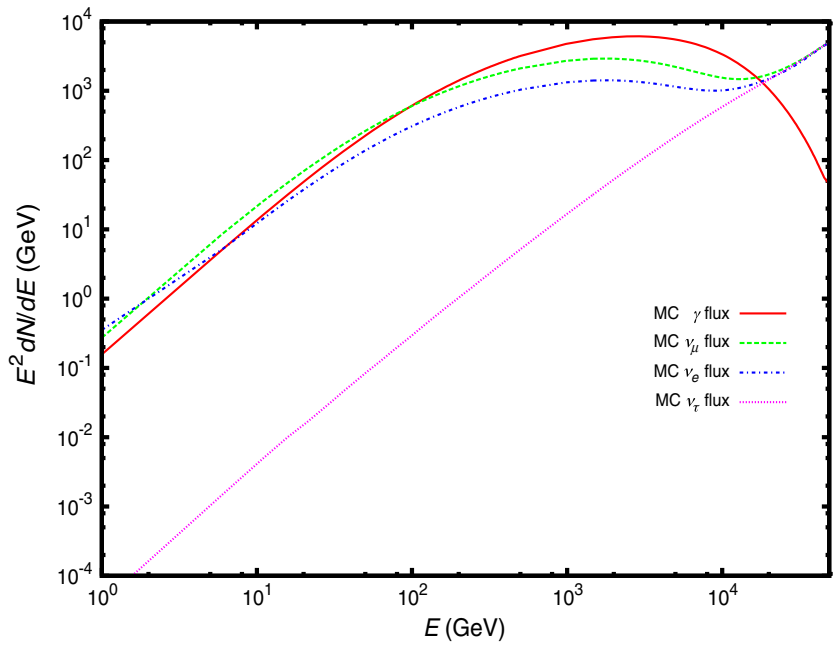

FIG. 1 (color online). The gamma-ray $(\gamma)$ and neutrino $\left(\nu_{p}\right)$ fluxes from $\mathrm{DM}$ of mass $M=48.8 \mathrm{TeV}$, annihilating into $W^{+} W^{-}$bosons, as generated by PYTHIA 8.135 and reported in Ref. [24]. 


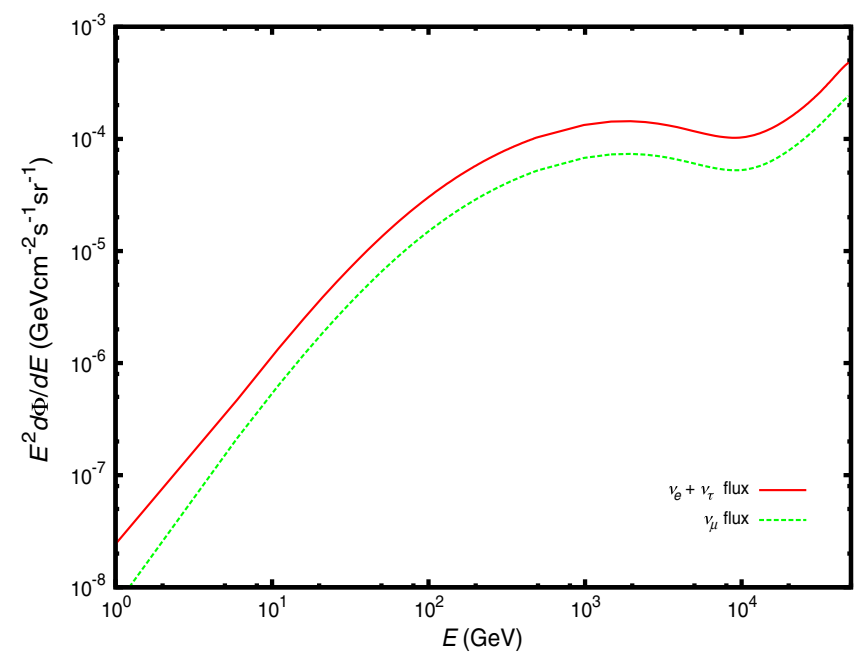

FIG. 2 (color online). Neutrino differential fluxes $\left(\Phi_{\nu_{e}}+\Phi_{\nu_{\tau}}\right.$ and $\left.\Phi_{\nu_{\mu}}\right)$ as expected to be observed at the Earth, taking into account both neutrino oscillations and the neutrinoantineutrino total flux. We are assuming DM annihilating into the $W^{+} W^{-}$channel. The parameters in Eq. (1) are $\langle\sigma v\rangle=3 \times 10^{-26} \mathrm{~cm}^{3} \mathrm{~s}^{-1}, \quad M=48.8 \mathrm{TeV}, \quad\left\langle J_{(2)}\right\rangle_{\Delta \Omega} \simeq 4.95 \times$ $10^{28} \mathrm{GeV}^{2} \mathrm{~cm}^{-5}$, and $\Delta \Omega=10^{-5}$.

easy to understand from the oscillation matrix (8) that the three flavors arrive at the Earth with very similar fluxes.

The differential number of neutrinos for the different flavors $\nu_{p}$, with $p=e, \mu$, and $\tau$, as generated by the Monte Carlo event generator software are shown in Fig. 1. The photon differential number is also shown for reference. As we have commented, the three flavors are produced with the same ratio at high energies, whereas the production of $\nu_{\tau}$ is negligible at low energies. In Fig. 2, we show the expected neutrino fluxes given by Eq. (1), as observed at the Earth, when oscillations and detection limits are taken into account. The parameters are given by the DM modelindependent fit of the HESS data with gamma rays characterized by Eq. (4) and $M \simeq 48.8 \mathrm{TeV}$ [11]. At this stage, the energy resolution of the neutrino detector has not yet been considered.

As we have commented, we are assuming that the neutrino detector will not be able to distinguish between neutrinos and antineutrinos [29]. So the neutrino flux $\Phi_{\nu_{\alpha}}$ is understood to be the sum of $\nu_{\alpha}$ and $\bar{\nu}_{\alpha}$. In addition, we shall assume that the detector will be able to distinguish muon neutrinos from electron and tau neutrinos. The latter flavors give a typical showering signal, whereas the $\nu_{\mu}$ provide a distinctive track signal. More precisely, neutrino flavors can be deduced from two different event topologies: muon tracks - related to the Cherenkov light of a propagating muon-and hadronic or electromagnetic showers. Showers are produced by neutral-current interactions of any neutrino flavor, and by both $\nu_{e}$ and $\nu_{\tau}$ charged-current (CC) interactions. On the other hand, tracks are induced by muons from $\nu_{\mu} \mathrm{CC}$ interactions and $\nu_{\tau} \mathrm{CC}$ interactions in which the tau decay produces a muon.

\section{ANALYSIS}

The most important source of background for highly energetic astrophysical neutrinos is given by atmospheric neutrinos and muons, depending on the direction of observation. The $\nu_{\mu}$ and $\nu_{e}$ atmospheric neutrinos have been reported by IceCube $[29,30]$. The electronic neutrino background has limited data with important uncertainties. In this case, the $\nu_{e}$ atmospheric flux can be well fitted by a simple power law,

$$
E^{2} \times \frac{d \Phi_{\nu_{e}}^{B g}}{d E}=A_{e}\left(\frac{E}{\mathrm{GeV}}\right)^{-B_{e}^{0}}
$$

with $A_{e}=0.012 \pm 0.011 \mathrm{GeVcm}^{-2} \mathrm{~s}^{-1} \mathrm{sr}^{-1}$ and $B_{e}^{0}=1.17$. IceCube has measured the muon neutrino background with more detail, and a modified power-law fitting function is needed to reproduce the observed data accurately,

$$
E^{2} \times \frac{d \Phi_{\nu_{\mu}}^{B g}}{d E}=A_{\mu}\left(\frac{E}{\mathrm{GeV}}\right)^{-\left(B_{\mu}^{0}+B_{\mu} \times \ln (E / \mathrm{GeV})\right)}
$$

with $A_{\mu}=0.05_{-0.02}^{+0.01} \mathrm{GeV} \mathrm{cm}^{-2} \mathrm{~s}^{-1} \mathrm{sr}^{-1}, B_{\mu}^{0}=0.81_{+0.008}^{-0.066}$, and $B_{\mu}=0.037$. The IceCube experimental data and both fitting functions within $1 \sigma$ standard deviation are shown in Fig. 3. The lack of $\nu_{e}$ atmospheric flux data and its large uncertainty allow for the power-law fit, but a decreasing

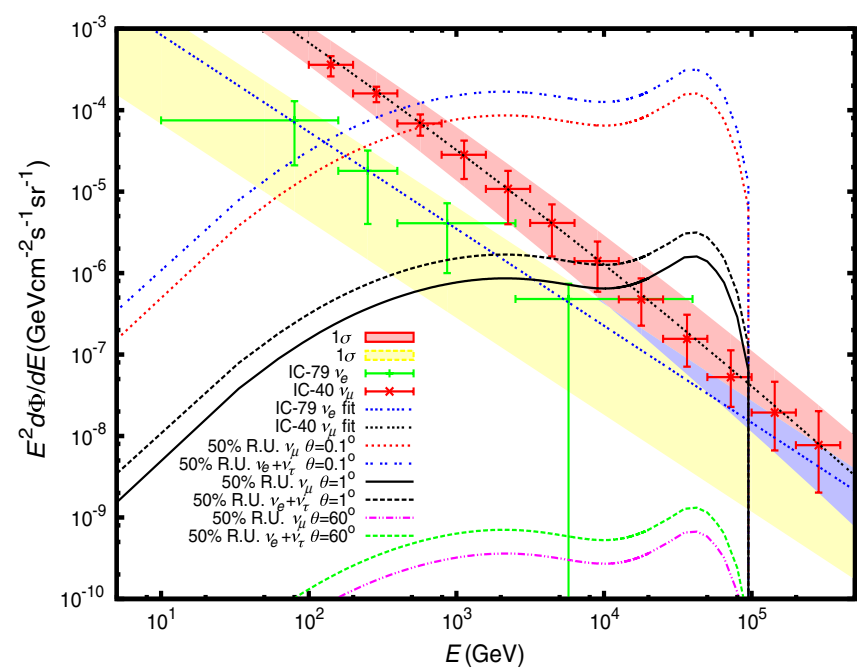

FIG. 3 (color online). Expected neutrino fluxes corresponding to muon neutrinos and electron plus tau neutrinos from DM annihilating into $W^{+} W^{-}$bosons for an angular field of view of $\theta=60^{\circ}, 1^{\circ}$, and $0.1^{\circ}$. The flux accounts for a $50 \%$ energy resolution uncertainty (R.U.) associated with a typical highenergy neutrino telescope. The atmospheric muons observed by the IceCube telescope in the 40-string configuration (IC-40) and electron neutrinos by the 79-string configuration (IC-79) are also shown together with the fitting functions given by Eqs. (10) and (9), respectively, and the corresponding shared regions are at the $1 \sigma$ confidence level. 
flux similar to the $\nu_{\mu}$ case is expected at energies higher than $10^{4} \mathrm{GeV}$. As we shall discuss, the analysis associated with the $\nu_{e}$ signal is not particularly interesting in this case due to its lower angular accuracy. Therefore, the overestimation of its atmospheric background at high energies does not have consequences in our results.

Our purpose is to estimate the possibilities that a general neutrino telescope will be sensitive to the neutrino signal associated to the HESS observation by assuming a DM origin. In order to be conservative, we will consider a $5 \sigma$ signal (or a less restrictive $3 \sigma$ or $2 \sigma$ confidence level) by comparing the number of events with respect to the atmospheric background for a particular neutrino signature,

$$
\chi_{\nu_{i}}=\frac{\Phi_{\nu_{i}} \sqrt{A_{\mathrm{eff}} t \times_{\mathrm{exp}} \Delta \Omega}}{\sqrt{\Phi_{\nu_{i}}+\Phi_{\nu_{i}}^{\mathrm{Atm}}}}=5(3,2),
$$

where the effective area $A_{\text {eff }}$, the solid angle $\Delta \Omega$, and the exposition time $t_{\text {exp }}$ depend on the particular detector and the observation. High-energy neutrino telescopes have an effective area in the $\mathrm{cm}^{2}$ to $\mathrm{km}^{2}$ range, depending not only on the experiment, but on the neutrino energy, the position of the source with respect to the telescope, and the associated type of background as well. We would like to note that Eq. (11) should be understood as a simple estimator, which contains important assumptions and simplifications. For example, it assumes an exposure that is constant in energy, and that the events follow a Poisson distribution. In addition, by selecting a priori an energy range for the analysis, it overestimates the significance of the detection. We can combine the track search and the shower signals in a common analysis.

However, we shall use only track events in our analysis since the angular resolution associated to the shower topology is much weaker. Indeed, high-energy muons point essentially in the same direction as the incident neutrino, since the angular resolution of high-energy muon tracks is quite good-smaller than $\theta=1^{\circ}$ for detectors as IceCube. This feature makes this topology particularly interesting for the analysis of DM annihilation in the GC. The electromagnetic or hadronic showers produced by neutrinos could be used as an additional signature to test the DM interpretation of the muon track signal. However, it is difficult to think that they can be used as the first evidence of DM neutrinos coming from the GC since (as we have commented) the current capabilities for shower angular resolution are much more limited.

For the IceCube/DeepCore detector, the GC is above the horizon, so the neutrino flux from this region contributes to the downward muon rate. However, for ANTARES [31] or the projected $\mathrm{KM} 3 \mathrm{NeT}$ [32] detector, the GC contributes to the upward muon rate. This fact is a clear advantage since the effective area and volume are enhanced.
As can be observed in Fig. 3, the sensitivity to DM in the GC depends crucially on the angular resolution. The best strategy consists in reducing the angle in order to decrease the atmospheric background. In such a case, an excess at energies of the order of $\sim 10 \mathrm{TeV}$ can be observable. In order to estimate the energy cutoff $E_{\min }^{\nu}$, we can restrict the total background to a few events: $\sum_{i=1}^{2} \Phi_{\nu_{i}}^{\text {Atm }} \times$ $A_{\text {eff }} t_{\text {exp }} \simeq 1$. As we have commented, we will assume that neutrinos produced by a point-like source are independent of the resolution angle of the neutrino telescope. In order to compute the number of neutrino events coming from DM, we integrate Eq. (1) over the observation time and energy,

$$
N_{\nu_{f}}^{t_{\mathrm{exp}}}=\int_{E_{\min }^{\nu}}^{\infty} d E_{\nu} \frac{d \Phi_{\nu_{f}}}{d E} \times A_{\mathrm{eff}} t_{\mathrm{exp}} .
$$

We shall not consider the probability of detecting a neutrino due to the proximity of its production to the detector. There is also an attenuation effect associated with neutrinos interacting within the Earth's volume [33,34]. It only affects to upward neutrinos and it shall be also neglected in our estimations. By fixing the exposition time $\left(t_{\text {exp }}=0.5,1,2,3,4\right.$, and 5 years in Figs. 4 and 8$)$, we can determine the minimum energy $E_{\min }^{\nu}$ that gives a certain

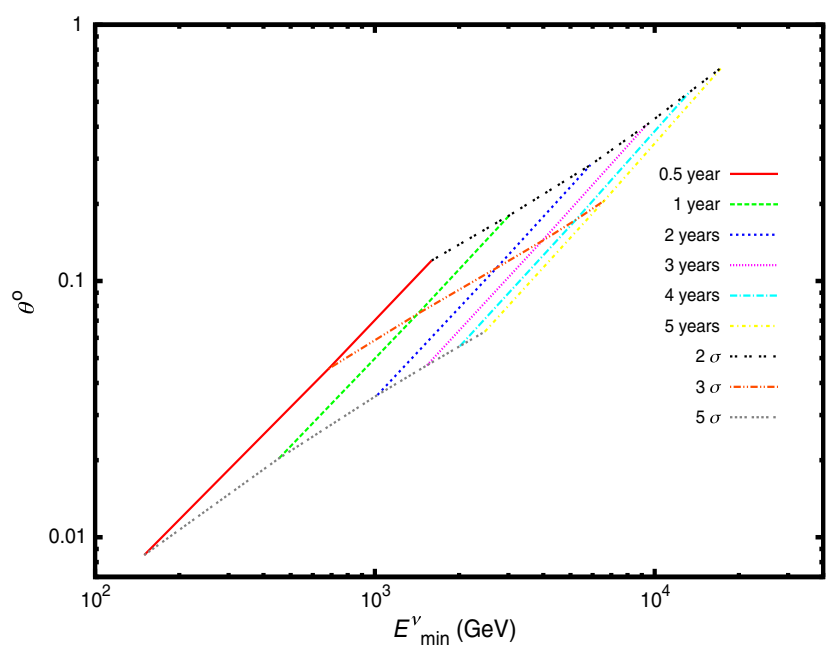

FIG. 4 (color online). Combination of the angular field of view $\theta$, minimum energy threshold, and exposition time that allow for the detection of a muon neutrino flux signal coming from DM annihilating in the $\mathrm{GC}$ at the $2 \sigma, 3 \sigma$, or $5 \sigma$ confidence levels, with a detector with $50 \mathrm{~m}^{2}$ effective area. The annihilating mode is the $W^{+} W^{-}$channel, the mass of the DM particles is $48.8 \mathrm{TeV}$, the annihilation cross section is $\langle\sigma v\rangle=3 \times 10^{-26} \mathrm{~cm}^{3} \mathrm{~s}^{-1}$, and the astrophysical factor is given by Eq. (4). The lowest value of $\theta \simeq$ $0.01^{\circ}$ corresponds to a $5 \sigma$ confidence level with an energy threshold of $E_{\min }^{\nu} \simeq 150 \mathrm{GeV}$ and 6 months of exposition time. The higher the exposition time, the higher the angular resolution of the analysis needed to reduce the atmospheric background. The largest value of $\theta \simeq 0.68^{\circ}$ is associated with 5 years of exposition time, a statistical significance of $2 \sigma$, and an energy threshold of $E_{\min }^{\nu} \simeq 17.42 \mathrm{TeV}$. 
number of neutrino events for each observation time (in the same figures, $N_{\nu_{\mu}} \simeq 25,9,4$, which are approximately associated with $5 \sigma, 3 \sigma$, or $2 \sigma$ if the background events are negligible). As opposed to the neutrino flux from DM, the events corresponding to the atmospheric background depend on the resolution angle of the telescope. For a given energy cut $E_{\min }^{\nu}$, we can find the maximum value for the angular field of view $\theta$ necessary to detect a negligible background (We have allowed one background event for the reported values in Figs. 4 and 8). We have developed qualitatively different analyses for two channels: the $W^{+} W^{-}$boson channel and $u \bar{u}$ quark-antiquark annihilation.

Following Ref. [11], DM annihilating into the $W^{+} W^{-}$ channel requires a DM mass of around $48.8 \mathrm{TeV}$ to fit the
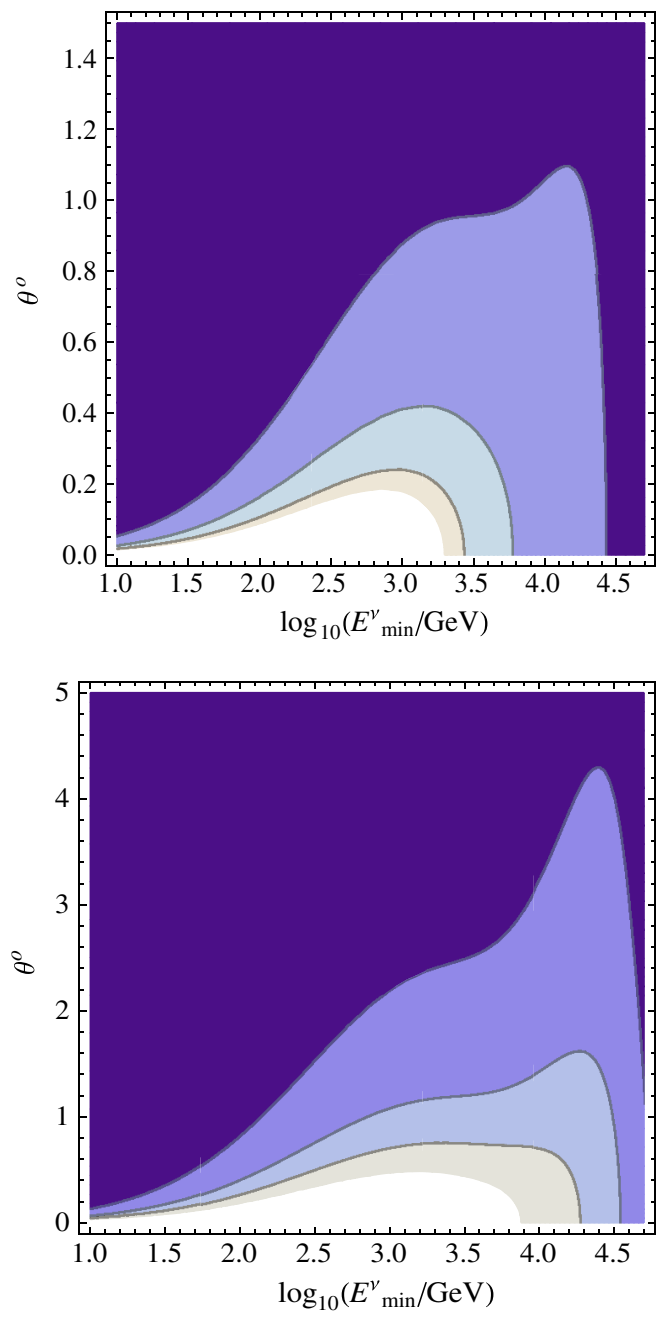

FIG. 5 (color online). The $1 \sigma$ (dark), $2 \sigma, 3 \sigma$, and $5 \sigma$ (white) confidence-level contours in the case of DM annihilating into the $W^{+} W^{-}$channel. The factor $A f=A_{\text {eff }} \times t_{\exp }$ is fixed in both analyses: $A f=100 \mathrm{~m}^{2} \mathrm{yr}$ (top panel) and $A f=600 \mathrm{~m}^{2} \mathrm{yr}$ (bottom panel). The possibility of detecting the neutrino flux signal above the atmospheric background depends on the energy cut $E_{\min }^{\nu}$ and the resolution angle.
HESS gamma-ray spectra of the J1745-290 source. As we can see in Fig. 3, no neutrino signal produced by this type of $\mathrm{DM}$ is expected with an angle of $\theta \approx 60^{\circ}$. In the same figure, it is shown that the DM flux can be observable for $\theta \sim 1^{\circ}$ or smaller (we are assuming a typical resolution energy of 50\%).

On the other hand, Figs. 5 and 6 are plotted without any constraint on the number of background events. The minimum energy thresholds for the $W^{+} W^{-}$channel are reported in Tables I and II for different effective areas and exposition times. We have studied the variation of the angular field of view and the energy cut. Larger sensitivities require very accurate angular resolutions. An analysis of energies larger than $E_{\min }^{\nu} \simeq 973 \mathrm{GeV}$ and an effective area of $A_{\text {eff }} \simeq 50 \mathrm{~m}^{2}$ with an exposition time of $t_{\mathrm{exp}} \simeq 5 \mathrm{yr}$ can provide a $5 \sigma$ detection signal for angular resolutions of $\theta \simeq 0.23^{\circ}$. Larger angular analyses of the order $\theta \simeq 0.7^{\circ}$
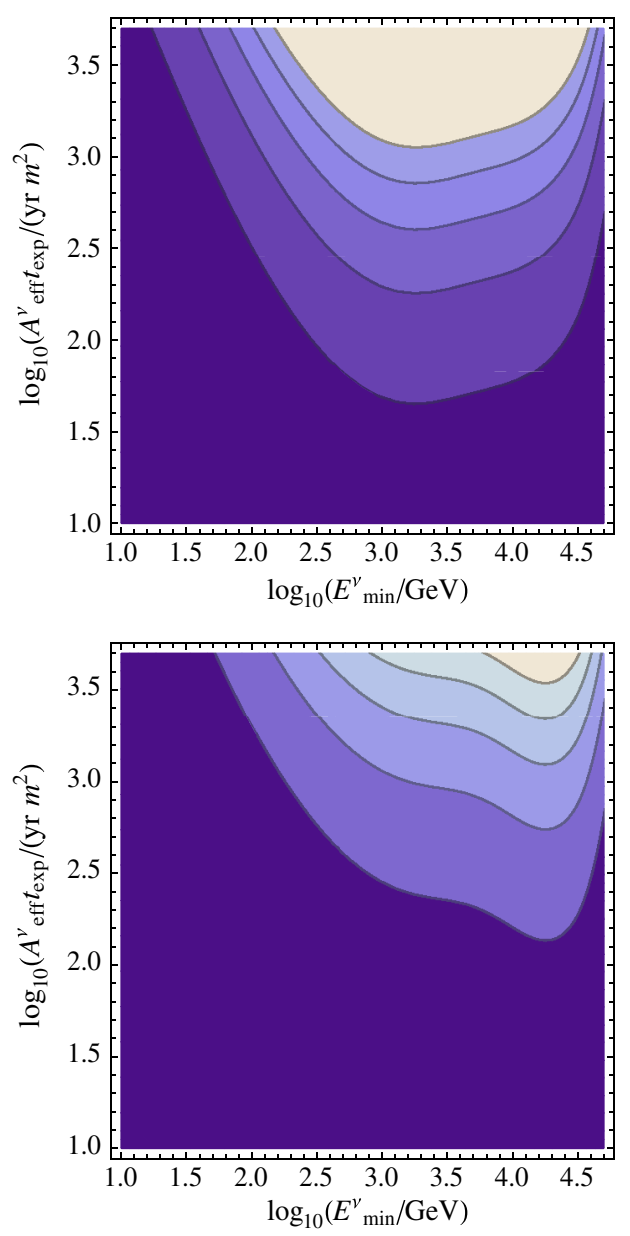

FIG. 6 (color online). As in Fig. 5, the $1 \sigma$ (dark), $2 \sigma, 3 \sigma, 4 \sigma$, and $5 \sigma$ (white) confidence-level contours for DM annihilating into the $W^{+} W^{-}$channel are plotted. In this case, the angular field of view is fixed as $\theta=0.6^{\circ}$ (top panel) and $\theta=1.5^{\circ}$ (bottom panel). Therefore, the possibility of detecting the neutrino flux signal above the atmospheric background depends on the energy cut $E_{\text {min }}^{\nu}$ and the factor $A f \equiv A_{\text {eff }} \times t_{\text {exp }}$. 
TABLE I. Energy threshold cut $(\mathrm{GeV})$ and resolution angle in order to achieve a confidence level of $5 \sigma, 3 \sigma$, or $2 \sigma$ from the muon neutrino flux for three different exposition times for DM annihilating into the $W^{+} W^{-}$channel with an effective area of $50 \mathrm{~m}^{2}$.

\begin{tabular}{lccc}
\hline \hline & \multicolumn{3}{c}{$E_{\min }^{\nu}(\mathrm{GeV})$} \\
\cline { 2 - 4 }$\theta^{\circ}$ & \multicolumn{3}{c}{$t_{\exp }$} \\
\cline { 2 - 4 } $5 \sigma$ & $2 \mathrm{yr}$ & $3 \mathrm{yr}$ & $5 \mathrm{yr}$ \\
\hline \multirow{3}{*}{$3 \sigma$} & 818 & 630 & 973 \\
& 0.18 & 0.15 & 0.23 \\
$2 \sigma$ & 977 & 1102 & 1737 \\
& 0.24 & 0.32 & 0.45 \\
& 1321 & 1482 & 1811 \\
\hline \hline
\end{tabular}

TABLE II. Same data reported in Table I but for an effective area of $5 \mathrm{~m}^{2}$.

\begin{tabular}{lccc}
\hline \hline & \multicolumn{3}{c}{$E_{\min }^{\nu}(\mathrm{GeV})$} \\
\cline { 2 - 4 } & & $t_{\exp }$ & \\
\cline { 2 - 4 }$\theta^{\circ}$ & $2 \mathrm{yr}$ & $3 \mathrm{yr}$ & $5 \mathrm{yr}$ \\
\hline $5 \sigma$ & & 21 & 156 \\
& & 0.002 & 0.003 \\
$3 \sigma$ & 110 & 176 & 334 \\
& 0.02 & 0.03 & 0.07 \\
$2 \sigma$ & 296 & 638 & 624 \\
& 0.06 & 0.15 & 0.15 \\
\hline \hline
\end{tabular}

can provide first evidence of these signatures with less statistical significance. In this case, the energy cut needs to be larger $\left(E_{\min }^{\nu} \simeq 18 \mathrm{TeV}\right)$ in order to reduce the atmospheric background. In Fig. 5, we show the resolution angle

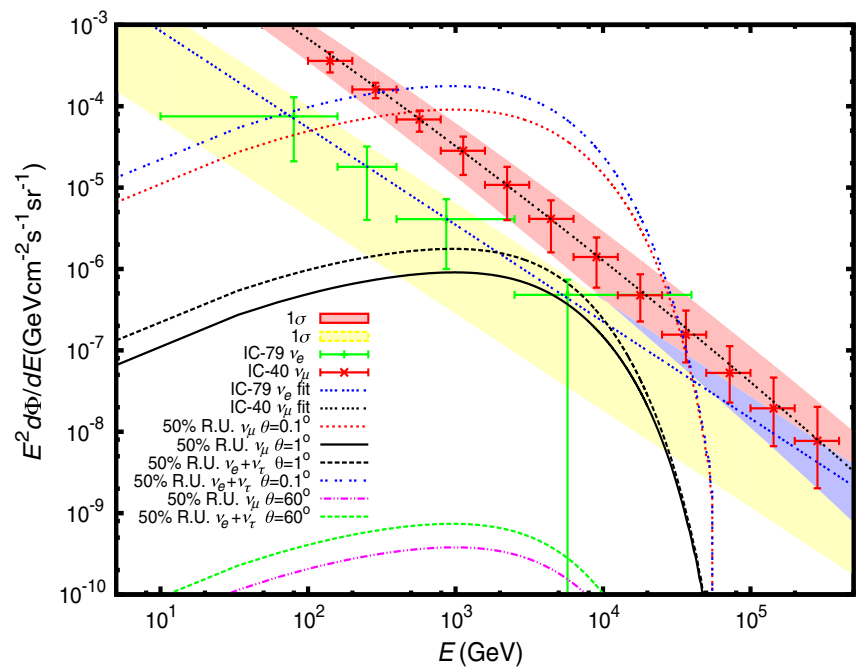

FIG. 7 (color online). Same information as in Fig. 3 but for DM annihilating into the $u \bar{u}$ channel.

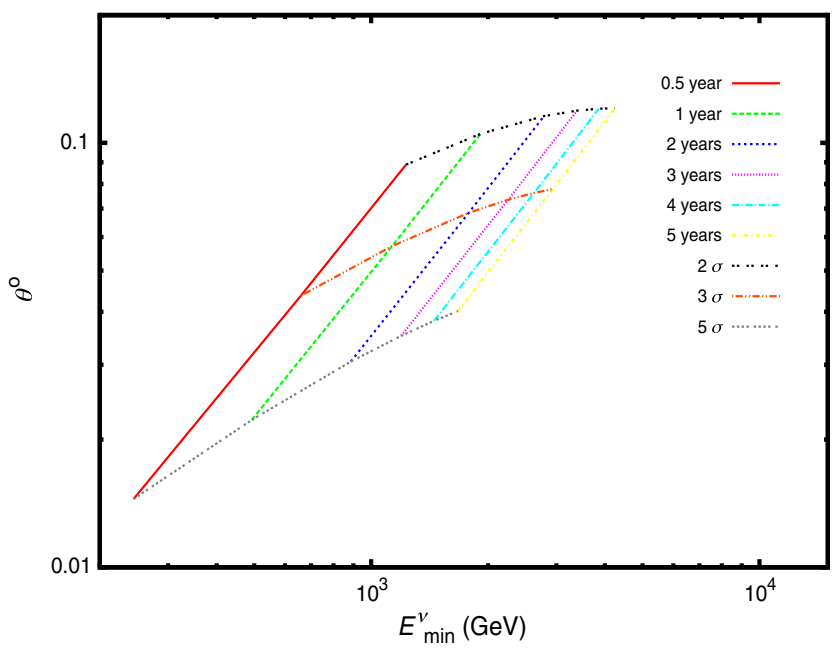

FIG. 8 (color online). Same information as in Fig. 4 but for the $u \bar{u}$ channel. In this case, the DM mass is fixed to $27.9 \mathrm{TeV}$, and the astrophysical factor is given by Eq. (5). The lowest value of $\theta \simeq 0.01^{\circ}$ corresponds to a $5 \sigma$ confidence level with an energy threshold of $E_{\min }^{\nu} \simeq 244 \mathrm{GeV}$ and 6 months of exposition time. The largest value of $\theta \simeq 0.12^{\circ}$ is associated with 5 years of exposition time, a statistical significance of $2 \sigma$, and an energy threshold of $E_{\min }^{\nu} \simeq 4.25 \mathrm{TeV}$.

$\theta$ as a function of the minimum energy cut $E_{\min }^{\nu}$ for different statistical significances and exposition times $t_{\exp }$. Similar information about the factor $A f \equiv A_{\text {eff }} \times t_{\exp }$ is given in Fig. 6.

The J1745-290 gamma-ray spectrum observed by HESS can also be well fitted by DM annihilating in hadronic modes. As an example, we have analyzed the $u \bar{u}$ quarkantiquark channel, which requires a mass close to $27.9 \mathrm{TeV}$ [11]. Under this assumption, we have repeated the study developed for the $W^{+} W^{-}$channel. In Fig. 7, we show the expected flux for different angular analyses. Estimations of the minimum energy cut and resolution angles depending on the exposition time and the statistical significance with negligible background are reported in Fig. 8. In Table III and Fig. 9, we present the results of the analysis for the same hadronic channel without constraining the number of

TABLE III. Same data reported in Table I but in the case of DM annihilating into the $u \bar{u}$ channel with an effective area of $50 \mathrm{~m}^{2}$.

\begin{tabular}{cccc}
\hline \hline & \multicolumn{3}{c}{$E_{\min }^{\nu}(\mathrm{GeV})$} \\
\cline { 2 - 4 }$\theta^{\circ}$ & $2 \mathrm{yr}$ & $3 \mathrm{yr}$ & $5 \mathrm{yr}$ \\
\cline { 2 - 4 } $5 \sigma$ & 274 & 336 & 420 \\
& 0.13 & 0.16 & 0.22 \\
$3 \sigma$ & 398 & 479 & 524 \\
& 0.24 & 0.30 & 0.40 \\
$2 \sigma$ & 490 & 839 & 552 \\
& 0.38 & 0.46 & 0.60 \\
\hline \hline
\end{tabular}



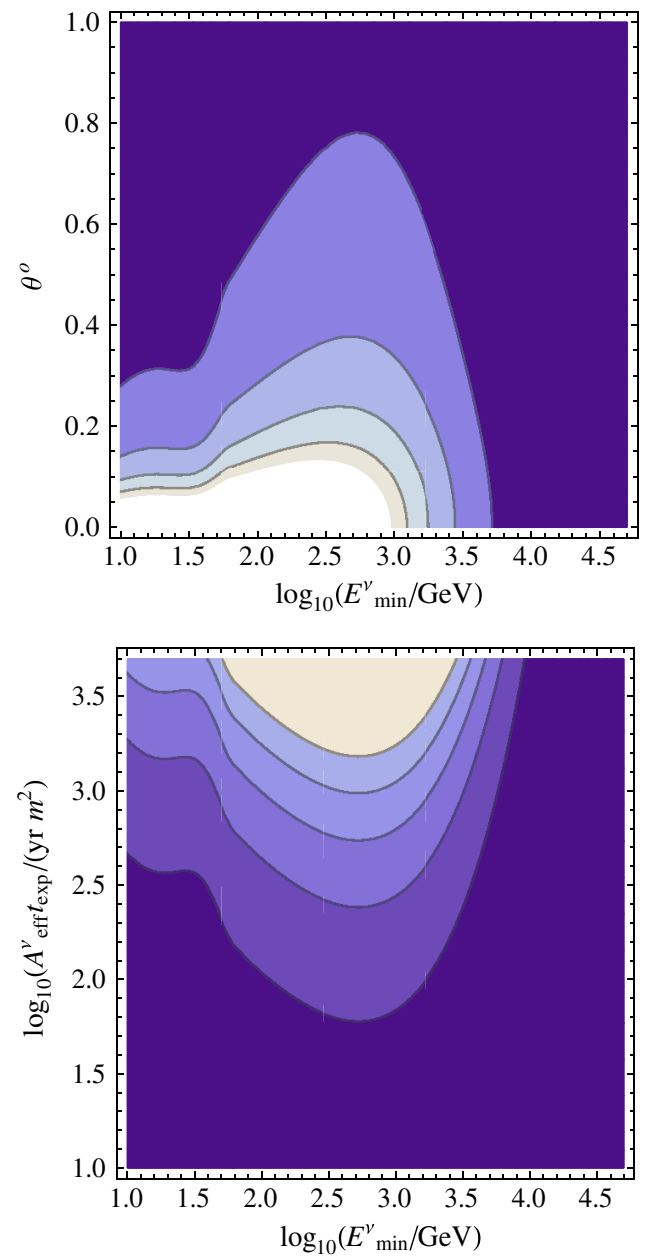

FIG. 9 (color online). Confidence-level contours associated with the observation of DM annihilating into the $u \bar{u}$ quarkantiquark channel at the $1 \sigma$ (dark), $2 \sigma, 3 \sigma, 4 \sigma$, and $5 \sigma$ (white) confidence levels. Top panel: The minimum energy cut is optimized around $1 \mathrm{TeV}$ depending on the resolution angle. The exposition time and effective area are fixed with the relation $A f \equiv A_{\text {eff }} \times t_{\text {exp }} \simeq 100 \mathrm{~m}^{2}$ yr. Bottom panel: The angular field of view is fixed at $\theta=0.6^{\circ}$. In such a case, the possibility of detecting the neutrino flux signal above the atmospheric background demands $A f \equiv A_{\mathrm{eff}} \times t_{\mathrm{exp}} \gtrsim 100 \mathrm{~m}^{2} \mathrm{yr}$.

background events, but we fix the combination of the effective area and exposition time $\left(A f=100 \mathrm{~m}^{2} \mathrm{yr}\right.$ in the upper panel) or the resolution angle $\left(\theta=0.6^{\circ}\right.$ in the lower panel).

\section{CONCLUSIONS}

The operation of the IceCube neutrino telescope at the South Pole, together with several counterparts in the Northern Hemisphere-such as ANTARES and NT200 (presently), or the future KM3NeT and GVD—are opening a new window onto our knowledge of neutrino astronomy.

Indeed, the construction of KM3NeT will imply a new substantial improvement in sensitivity corresponding to a $\mathrm{km}^{3}$ sized detector. On the other hand, radio and airshower detectors, such as ANITA and the Pierre Auger Observatory, are sensitive to neutrinos with even higher energies. The development of neutrino detectors have increased the interest for analyzing the nature of DM through the production of astrophysical neutrinos as its primary source.

We have studied the prospective neutrino fluxes that should originate from DM annihilating in the GC, in the case that the J1745-290 HESS high-energy gamma rays have this origin. As was shown in Ref. [11], a powerlaw spectrum is not consistent with the HESS data $\left(\chi^{2} /\right.$ d.o.f. $\left.=2.48\right)$ but there is not a statistically significant difference between a broken power law $\left(\chi^{2} /\right.$ d.o.f. $\left.=0.87\right)$ and the DM annihilation hypothesis. Indeed, the photon spectra is well fitted by different electroweak $\left(\chi^{2} /\right.$ d.o.f. $=$ 0.84 for $\left.W^{+} W^{-}\right)$and hadronic $\left(\chi^{2} /\right.$ d.o.f. $=0.78$ for $\left.u \bar{u}\right)$ channels. We have done an explicit analysis for $48.8 \mathrm{TeV}$ DM annihilating into $W^{+} W^{-}$and 27.9 TeV DM annihilating into the $u \bar{u}$ channel. In these cases, the neutrino fluxes are completely determined by assuming that the DM region is localized, as is imposed by the gamma-ray analysis. We have estimated the best combinations of energy cuts, observation times, and angular resolutions of a general high-energy neutrino telescope.

For this purpose, we have used IceCube atmospheric neutrino observations as background. In particular, the data were collected with an exposition time of $t_{\exp }^{\nu_{\mu}}=359$ days and $t_{\exp }^{\nu_{e}}=281$ days for the muon and electron neutrinos, respectively $[29,30]$. We have found that for DM annihilating into the $W^{+} W^{-}$boson channel, we need a resolution angle $0.18^{\circ} \lesssim \theta \lesssim 0.72^{\circ}$ and low-energy cutoff $818 \mathrm{GeV} \lesssim E_{\min }^{\nu} \lesssim 1811 \mathrm{GeV}$ to get a signal between $5 \sigma$ and $2 \sigma$ with a minimum of 2 years of exposition time and a maximum of 5 years for an effective detector area of $50 \mathrm{~m}^{2}$. The mass associated with the $u \bar{u}$ annihilation channel is significantly smaller. This implies that the neutrino flux produced in this case is less energetic and more difficult to discriminate from the background. It demands a higher angular resolution $\left(0.13^{\circ} \lesssim \theta \lesssim 0.60^{\circ}\right)$ and the energy cuts need to be smaller $(274 \mathrm{GeV} \lesssim$ $E_{\min }^{\nu} \lesssim 552 \mathrm{GeV}$ ) in order to accumulate enough events. We have considered only track signal data by rejecting the muon background and taking into account the total number of events. For a binned analysis with a nonzero background and with a combined analysis of track and shower signatures, it could be possible to find better experimental configurations that should allow for the detection of neutrinos produced by heavy DM from the $\mathrm{GC}$ with a worse resolution angle, smaller effective area, or less exposition time.

This DM interpretation is compatible with other cosmicray constraints (such as antiprotons or radio and $\mathrm{x}$-ray frequencies). The main reason for this is that the sensitivity of these analyses decreases with the DM mass and the 
HESS data demands a heavy DM particle. In addition, these other searches depend on the total distribution of the DM halo, which introduces important uncertainties into the study.

Recently, the IceCube Collaboration have reported the observation of 37 extraterrestrial high-energy neutrinos over the range $30 \mathrm{TeV}-2 \mathrm{PeV}$ at the $5.7 \sigma$ of confidence level, and $t_{\exp }=988$ days [34]. There is not clear statistical evidence of clustering or spatial correlations (although the strongest clustering is near the Galactic center). These neutrinos seem to have an astrophysical origin, but the spectrum and spatial distribution are not compatible with the signal studied in this work (the angular resolution in the muon track events is of $\theta \lesssim 1^{\circ}$ ). The DM signal analyzed in this work may only account for a small part of the events, which will is more likely associated with an electroweak channel, such as the $W^{+} W^{-}$annihilating DM model.
In any case, we would like to remark that the detection of a neutrino emission from the J1745-290 source cannot be taken as a confirmation of the DM nature of the signal since a different origin may produce such events. However, if the spectral features of the neutrino flux are consistent with the DM prediction, it can be an important indication in favor of the DM hypothesis.

\section{ACKNOWLEDGMENTS}

We would like to thank Juande Zornoza and Carlos de los Heros for useful comments. This work has been supported by UCM predoctoral grant, MICINN (Spain) project numbers FIS 2008-01323, FIS2011-23000, FPA2011-27853-01, Consolider-Ingenio MULTIDARK CSD2009-00064, and the Department of Energy, Contract DE-AC02-76SF00515. J. A. R. C. thanks the support of the Becas Complutense del Amo program.
[1] K. Tsuchiya et al., Astrophys. J. 606, L115 (2004).

[2] K. Kosak et al., Astrophys. J. 608, L97 (2004).

[3] J. Albert et al., Astrophys. J. 638, L101 (2006).

[4] V. Vitale and A. Morselli (for the Fermi/LAT Collaboration), arXiv:0912.3828.

[5] M. Cherenyakova, D. Malyshev, F. A. Aharonian, R. M. Crocker, and D. I. Jones, Astrophys. J. 726, 60 (2011); T. Linden, E. Lovegrove, and S. Profumo, Astrophys. J. 753, 41 (2012).

[6] F. Aharonian et al., Astron. Astrophys. 425, L13 (2004).

[7] F. Aharonian et al., Astron. Astrophys. 503, 817 (2009).

[8] Q. Wang, F. Lu, and E. Gotthelf, Mon. Not. R. Astron. Soc. 367, 937 (2006); B. Aschenbach, N. Grosso, D. Porquet, and P. Predehl, Astron. Astrophys. 417, 71 (2004).

[9] L. Bergstrom, T. Bringmann, M. Eriksson, and M. Gustafsson, Phys. Rev. Lett. 94, 131301 (2005); Phys. Rev. Lett. 95, 241301 (2005).

[10] F. Aharonian et al., Phys. Rev. Lett. 97, 221102 (2006).

[11] J. A. R. Cembranos, V. Gammaldi, and A. L. Maroto, Phys. Rev. D 86, 103506 (2012); J. Cosmol. Astropart. Phys. 04 (2013) 051.

[12] J. Cohen-Tanugi et al., in Proceedings of The 31st International Cosmic Ray Conference, Lodz, Poland, 7-15 July, 2009, http://icrc2009.uni.lodz.pl/proc/pdf/ icrc0645.pdf.

[13] R. M. Crocker, M. Fatuzzo, J. R. Jokipii, F. Melia, and R. R. Volkas, Astrophys. J. 622, 892 (2005).

[14] A. Atoyan and C. D. Dermer, Astrophys. J. 617, L123 (2004).

[15] F. Aharonian and A. Neronov, Astrophys. J. 619, 306 (2005).

[16] J. F. Navarro, C. S. Frenk, and S. D. White, Astrophys. J. 490, 493 (1997).
[17] G. R. Blumenthal, S. M. Faber, R. Flores, and J. R. Primack, Astrophys. J. 301, 27 (1986); O. Y. Gnedin, A. V. Kravtsov, A. A. Klypin, and D. Nagai, Astrophys. J. 616, 16 (2004).

[18] F. Prada, A. Klypin, J. Flix Molina, M. Martínez, and E. Simonneau, Phys. Rev. Lett. 93, 241301 (2004).

[19] E. Romano-Díaz, I. Shlosman, Y. Hoffman, and C. Heller, Astrophys. J. 685, L105 (2008); Astrophys. J. 702, 1250 (2009); A. V. Maccio', G. Stinson, C. B. Brook, J. Wadsley, H. M. P. Couchman, S. Shen, B. K. Gibson, and T. Quinn, Astrophys. J. 744, L9 (2012).

[20] J. Alcaraz, J. Cembranos, A. Dobado, and A. Maroto, Phys. Rev. D 67, 075010 (2003); P. Achard et al., Phys. Lett. B 597, 145 (2004); J. A. R. Cembranos, A. Dobado, and A. L. Maroto, Phys. Rev. D 65, 026005 (2001); Phys. Rev. D 70, 096001 (2004); 73, 035008 (2006); 73, 057303 (2006); J. Phys. A 40, 6631 (2007); J. A. R. Cembranos, J. L. Diaz-Cruz, and L. Prado, Phys. Rev. D 84, 083522 (2011); J. A. R. Cembranos, R. L. Delgado, and A. Dobado, Phys. Rev. D 88, 075021 (2013).

[21] J. A. R. Cembranos, J. L. Feng, and L. E. Strigari, Phys. Rev. Lett. 99, 191301 (2007); J. A. R. Cembranos and L. E. Strigari, Phys. Rev. D 77, 123519 (2008); J. A. R. Cembranos, A. de la Cruz-Dombriz, V. Gammaldi, and A. L. Maroto, Phys. Rev. D 85, 043505 (2012).

[22] T. Sjostrand, S. Mrenna, and P. Skands, J. High Energy Phys. 05 (2006) 026.

[23] J. A. R. Cembranos, A. de la Cruz-Dombriz, A. Dobado, R. Lineros, and A. L. Maroto, Phys. Rev. D 83, 083507 (2011); AIP Conf. Proc. 1343, 595 (2011); J. Phys. Conf. Ser. 314, 012063 (2011); A. de la Cruz-Dombriz and V. Gammaldi, arXiv:1109.5027.

[24] M. Cirelli, G. Corcella, A. Hektor, G. Htsi, M. Kadastik, P. Panci, M. Raidal, F. Sala, and A. Strumia, J. Cosmol. Astropart. Phys. 03 (2011) 051. 
[25] L. Sadeghian, F. Ferrer, and C. M. Will, Phys. Rev. D 88, 063522 (2013).

[26] R. M. Crocker, F. Melia, and R. R. Volkas, arXiv:astro-ph/ 9911292v2; N. F. Bell, J. Phys. Conf. Ser. 136, 022043 (2008).

[27] K. C. Lai, G. L. Lin, and T. C. Liu, Phys. Rev. D 80, 103005 (2009).

[28] J. Beringer et al. (Particle Data Group), Phys. Rev. D 86, 010001 (2012).

[29] M. G. Aartsen et al. (IceCube Collaboration), Phys. Rev. Lett. 110, 151105 (2013).

[30] R. Abbasi et al. (IceCube Collaboration), Phys. Rev. D 83, 012001 (2011); M. G. Aartsen et al. (IceCube Collaboration), Astrophys. J. 779, 132 (2013).

[31] S. Adrian-Martinez et al. (ANTARES Collaboration), Astrophys. J. 760, 53 (2012); S. Schulte (for the ANTARES Collaboration), in Proceedings of The 33rd International Cosmic Ray Conference, Rio de Janeiro, Brazil, 2-9 July,
2013, http:/www.cbpf.br/ icrc2013/papers/icrc2013-0425 .pdf.

[32] T. Seitz and R. Shanidze (KM3NET Consortium), Nucl. Instrum. Methods Phys. Res., Sect. A 626-627, S205 (2011).

[33] R. M. Crocker, F. Melia, and R. R. Volkas, arXiv:astro-ph/ 0411471.

[34] M. G. Aartsen et al. (IceCube Collaboration), arXiv: 1405.5303 [Phys. Rev. Lett (to be published)]; N. Whitehorn, C. Kopper, and N. K. Neilson (for the IceCube Collaboration), in Proceedings of the IceCube Particle Astrophysics Symposium 2013, Madison, Wisconsin, USA, 13-15 May, 2013 (to be published); F. Halzen, S. Klein, and C. Kopper, in Proceedings of The 33rd International Cosmic Ray Conference, Rio de Janeiro, Brazil, 2-9 July, 2013, http://www.cbpf.br/icrc2013; D. B. Fox, K. Kashiyama, and P. Meszaros, Astrophys. J. 774, 74 (2013); S. Razzaque, Phys. Rev. D 88, 081302 (2013). 